\title{
Photometric Search for Black Holes in Dormant X-Ray Transients
}

\author{
Aleksander Sądowski \\ Astronomical Observatory of Warsaw University, Warsaw, Poland \\ E-mail: oleks@camk.edu.pl \\ Janusz Ziolkowski* \\ Copernicus Astronomical Center, Warsaw, Poland \\ E-mail: jz@camk.edu.pl
}

\section{Krzysztof Belczyński}

Department of Astronomy, New Mexico State University, Las Cruces, New Mexico, USA

E-mail: belczynski@northwestern.edu

\begin{abstract}
Our calculations of stellar population synthesis indicate that the Galaxy contains some 15000 to 40000 dormant X-ray transients with a black hole component. It means that there should be some 20 to 50 such systems within $0.5 \mathrm{kpc}$ from the Sun. For dormant X-ray transients with a neutron star component the corresponding numbers are 30000 to 250000 in the Galaxy and 40 to 300 in the solar vicinity. These objects do not emit $\mathrm{X}$ rays, but their optical components should display ellipsoidal light variations, typical for Roche lobe filling components of close binaries. For a typical soft X-ray transient (mass of the optical component 0.5 to $2 \mathrm{M}_{\odot}$, mass of the compact component 5 to $10 \mathrm{M}_{\odot}$ (black hole) or $1.5 \mathrm{M}_{\odot}$ (neutron star)), the amplitudes of these variations (in $V$ band) should be up to 0.5 magnitudes (depending on the inclination of the system). Such variability should be easy to detect in mass photometric surveys (such as ASAS).
\end{abstract}

VI Microquasar Workshop: Microquasars and Beyond

September 18-22, 2006

Como, Italy

${ }^{*}$ Speaker. 


\section{Introduction}

It is well known that majority of black hole (BH) X-ray binaries are transient sources. Among the presently known some 50 black hole candidate (BHC) systems, about $80 \%$ are transient X-ray sources. Due to selection effects, the fraction of transient systems is, probably, even higher. The transient systems for most of the time are dormant, i.e. they do not emit $\mathrm{X}$ rays. However, their optical components fill their Roche lobes all the time (also during the dormant phase). Therefore, they should display the ellipsoidal light variations, typical for tidally distorted stars. Synthetic light curves, calculated for typical such systems (mass of the optical component $0.6 \mathrm{M}_{\odot}$, mass of the compact component $6 \mathrm{M}_{\odot}$ (black hole) or $1.5 \mathrm{M}_{\odot}$ (neutron star)) by Dymitrow [3], indicate that the amplitudes of these variations (in $V$ band) should be up to 0.5 magnitude (depending on the inclination of the system).

We carried out the calculations of stellar population synthesis in order to estimate the number of transient BH X-ray binaries in the Galaxy. The aim of this estimate was to find what are the chances of detecting these ellipsoidal light variations in mass photometric surveys.

The calculations were carried out using the Star Track code described by Belczyński, Kalogera \& Bulik [1] and Belczyński et al. [2]. We define BH X-ray binary as a system composed of a black hole and an optical star filling its Roche lobe (in all BH systems known, the optical component practically fills its Roche lobe, even if $\mathrm{BH}$ is accreting from the stellar wind). Such definition means that our statistics for the number of expected BH X-ray binaries include both transient and persistent systems. However, as it was pointed earlier, great majority of these systems must be transient sources.

\section{Calculations}

The results of calculations of stellar population synthesis are sensitive to the values of different parameters entering the code and to the different assumption made during the calculations. We found that, in the calculations carried out for our purposes, the most critical parameter was the assumed value of the critical mass of the final compact object $M_{\mathrm{cr}}$, below which it is a neutron star (NS) and above which it is a BH. Therefore, we carried out all calculations for two values of $M_{\mathrm{cr}}: 2$ $\mathrm{M}_{\odot}$ and $3 \mathrm{M}_{\odot}$. We believe, that the lower value is more correct. The distribution of the determined masses of NSs covers the range from $1.25 \mathrm{M}_{\odot}$ to about $2 \mathrm{M}_{\odot}$ - see, e.g., Ziółkowski [8]. However, this argument is not conclusive enough to rule out the higher value. Therefore, for each of the two values of $M_{\text {cr }}$ mentioned above, we performed calculations, assuming relatively wide ranges for other parameters entering the numerical code. The resulting distributions of orbital periods of $\mathrm{BH}$ $\mathrm{X}$-ray binaries were compared with the observed distribution to select the plausible values of these parameters.

\section{Preliminary Results}

Fig. 1 shows the results of the calculations made with the standard values of the parameters and standard assumptions used in the Star Track code. The red line shows the observed distribution of orbital periods of BH X-ray binaries based on catalogs of Liu et al. [4], [5]. 
$M_{C R}=2 M_{S U N}$

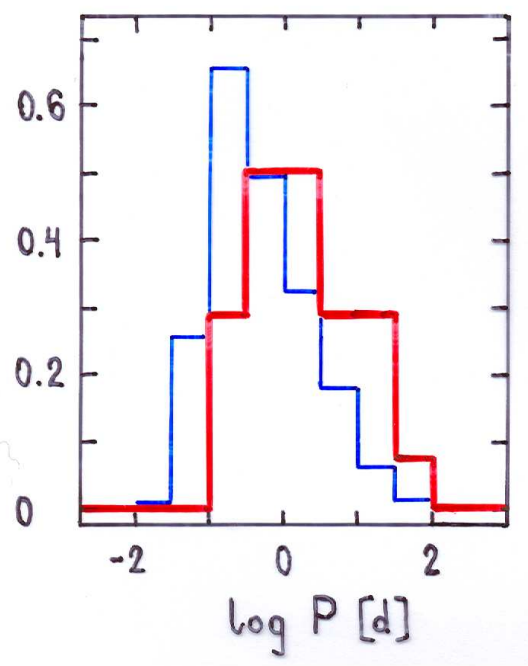

$M_{C R}=3 M_{S U N}$

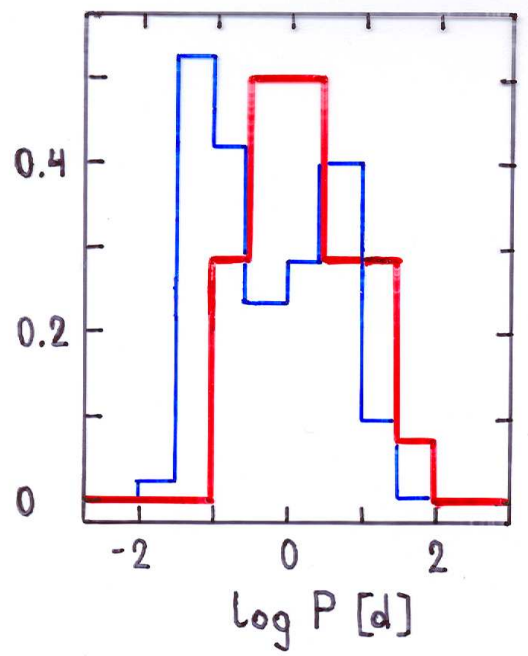

Figure 1: Observed (red line) and expected (blue line) distributions of orbital periods of BH X-ray binaries for two values of the parameter $M_{\mathrm{cr}}$ (see the text). The observed distribution is based on catalogs of Liu et al. [4], [5]. The expected one is the result of stellar population synthesis calculations with standard values of all parameters.

One may notice that the value $M_{\mathrm{cr}}=2 \mathrm{M}_{\odot}$ produces better fit to the observations than the value $M_{\mathrm{cr}}=3 \mathrm{M}_{\odot}$.

$$
M_{C R}=2 M_{S U N}
$$$$
M_{C R}=3 M_{S U N}
$$
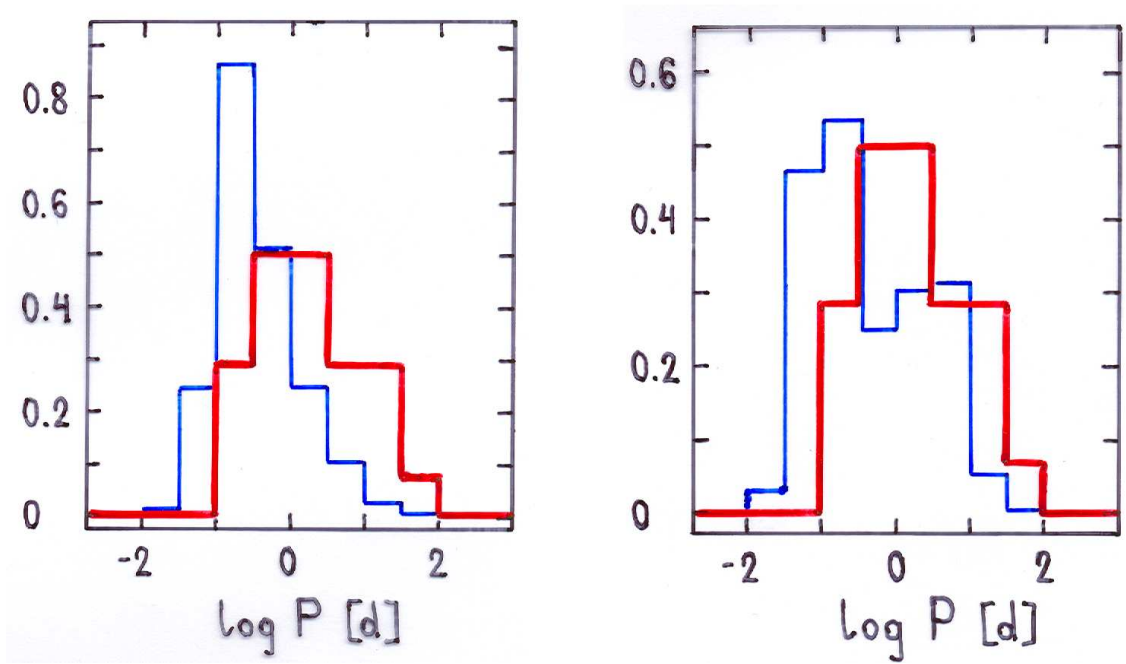

Figure 2: The same as Fig.1 but for the value of the parameter describing the efficiency of the common envelope reduced by a factor of 10 (see the text).

Fig. 2 shows the results obtained when one changes the assumption concerning the efficiency 
of the process of the shedding of the envelope during the common envelope phase of the stellar evolution. One of the parameters used in the code describes how efficiently the gravitational energy released due to shrinking of the binary orbit (the orbit shrinks because both components experience drag force while moving in the common envelope) is used to unbind the common envelope.

Here the value of this parameter was decreased by a factor of 10 with respect to the standard value.

As one may see, the fit is now worse than in Fig. 1 (the fit for $M_{\mathrm{cr}}=2 \mathrm{M}_{\odot}$ still being much better than the fit for $M_{\mathrm{cr}}=3 \mathrm{M}_{\odot}$ ).

$$
\mathrm{M}_{\mathrm{CR}}=2 \mathrm{M}_{\mathrm{SUN}}
$$

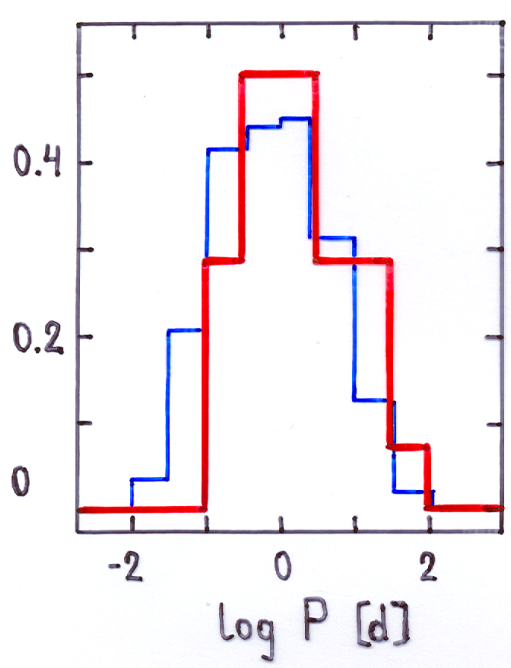

$$
\mathrm{M}_{\mathrm{CR}}=3 \mathrm{M}_{\mathrm{SUN}}
$$

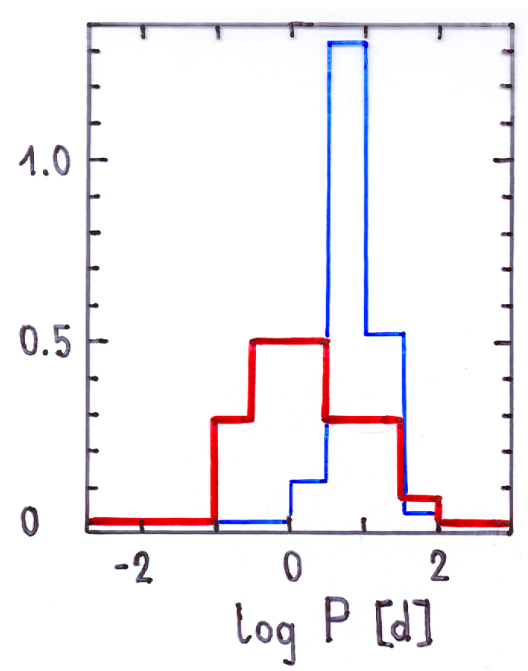

Figure 3: The same as Fig.1 but for the assumption of no kick during the collapse event producing a black hole (see the text).

Fig. 3 shows the distribution expected if one assumes that black holes are produced with no kick during the collapse event. The standard approach assumes that the distribution of the kicks is the same as for the events producing neutron stars (this distribution is inferred from the space velocities of radio pulsars). There are, however, some observational arguments that many black holes may be produced with no substantial kicks (either through a dark collapse or with only small mass ejection, as demonstrated by Mirabel and Rodrigues [6]).

This case produces the best fit so far for $M_{\mathrm{cr}}=2 \mathrm{M}_{\odot}$ (for $M_{\mathrm{cr}}=3 \mathrm{M}_{\odot}$ the fit is very poor).

Fig. 4 shows the same case as Fig. 3 (for $M_{\mathrm{cr}}=2 \mathrm{M}_{\odot}$ ), but with the final systems composed of black hole and white dwarf being removed from the expected population. This removal is quite arbitrary. It is true that we do not observe such systems, but they are produced in the stellar population synthesis calculations and we do not understand what is the reason for this discrepancy.

As may be seen, the removal of black hole + white dwarf binaries improves the fit (the green line in Fig. 4). 


$$
M_{C R}=2 M_{S U N}
$$

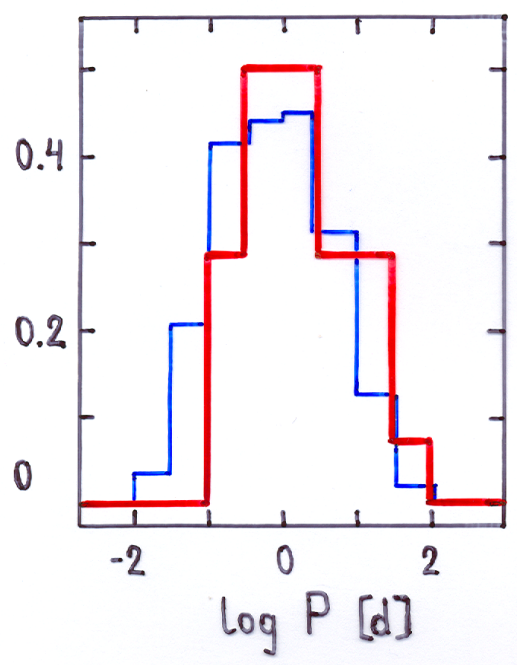

$M_{C R}=3 M_{S U N}$

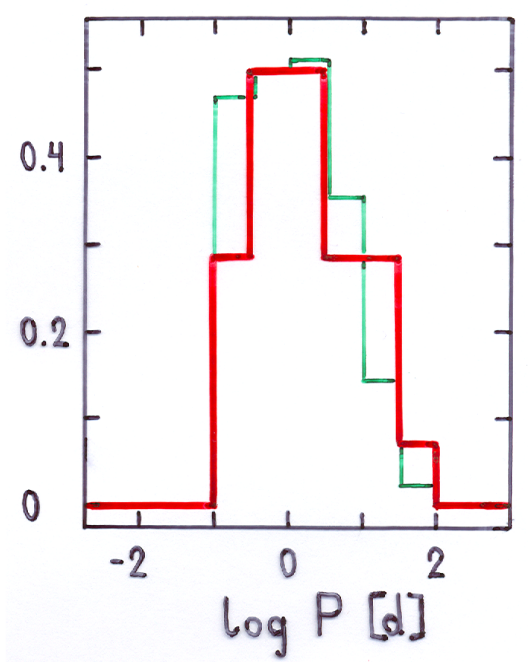

Figure 4: The same as Fig. 1 but for one value of $M_{\mathrm{cr}}$ parameter $\left(M_{\mathrm{cr}}=2 \mathrm{M}_{\odot}\right)$. The left panel is the same as in Fig. 3 (no kick case). The right panel is the same as the left panel, but with the black hole + white dwarf binaries arbitrarily removed from the expected population (the green line - see the text).

\section{Population of BH X-Ray Binaries}

The expected numbers of galactic BH X-ray binaries depends mainly on the value of the parameter $M_{\mathrm{cr}}$. If this value is $2 \mathrm{M}_{\odot}$, then, according to our calculations, Galaxy contains some 15000 to 40000 of BH X-ray binaries (and some 30000 to 250000 of NS X-ray binaries). If this value is $3 \mathrm{M}_{\odot}$, then our Galaxy contains 800 to 6000 of BH X-ray binaries (and some 70 000 to 250000 of NS X-ray binaries). As we have seen (Section 3), the $2 \mathrm{M}_{\odot}$ value produces, systematically, substantially better fits of the expected orbital period distributions to the observed one. There are also some other arguments favoring the lower value (see Section 2). However, as it was noted earlier, the evidence is not conclusive enough to rule out the higher value. Therefore, the range of the expected number of galactic $\mathrm{BH} X$-ray binaries remains uncomfortably wide (between 800 and 40000$)$. We may add that, using the most likely set of assumptions $\left(M_{\mathrm{cr}}=2 \mathrm{M}_{\odot}\right.$, no kick during $\mathrm{BH}$ formation), we obtain about 15000 for the expected number of BH X-ray binaries.

\section{Photometric search for BHs in Dormant X-Ray Transients}

Fig. 5 shows the synthetic light curves reflecting the ellipsoidal variability (in $V$ band) of a dormant (no X-ray irradiation effects) X-ray binary. The left panel shows the variability of a BH $\mathrm{X}$-ray binary and the right panel the variability of a NS X-ray binary. The assumed masses were: $6 \mathrm{M}_{\odot}$ for a black hole, $1.5 \mathrm{M}_{\odot}$ for a neutron star and $0.6 \mathrm{M}_{\odot}$ for a lower main sequence optical component. The orbital periods were adjusted so that the optical components fill, in both cases, their Roche lobes. The pictures describe the case of edge-on $\left(i=90^{\circ}\right)$ orientation of the binary orbit. The light curves were computed by Dymitrow [3]. 

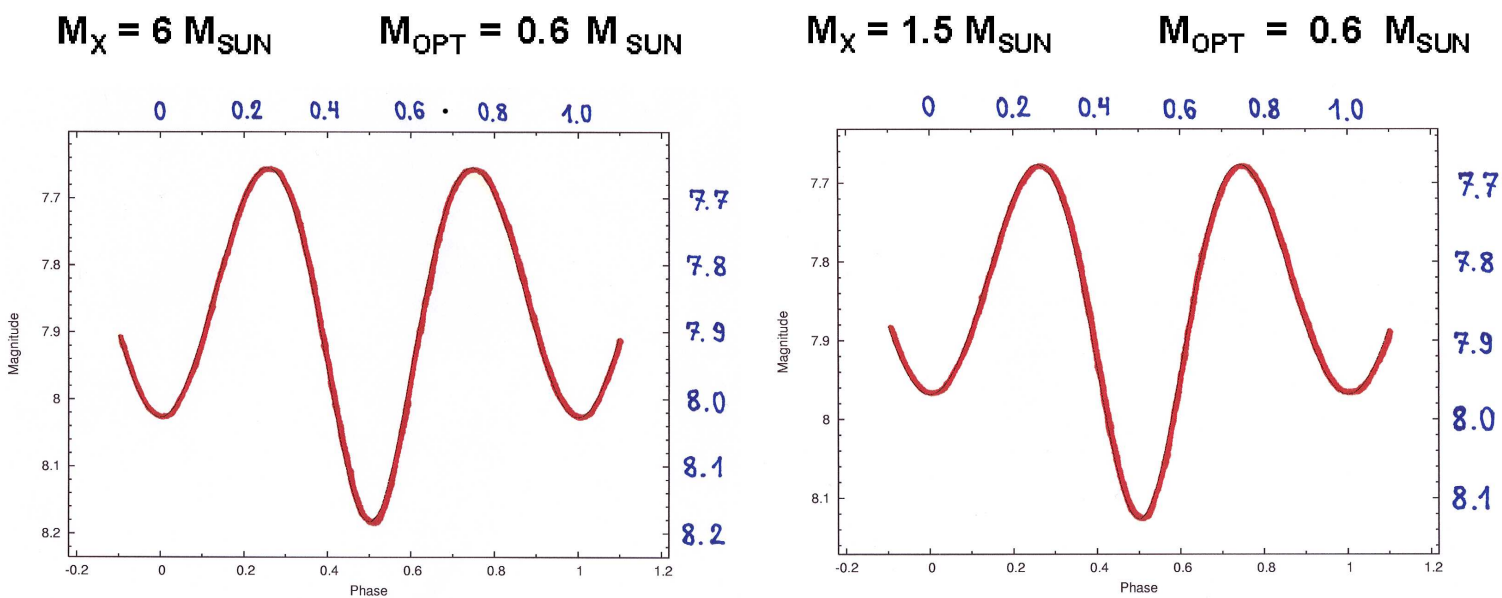

Figure 5: The synthetic light curves showing the ellipsoidal variability (in $V$ band) of dormant (no $\mathrm{X}$-ray irradiation effects) X-ray binaries. The left panel shows the variability of a BH X-ray binary and the right panel the variability of a NS X-ray binary. The assumed masses of the components are given above the panels. The orbital periods were adjusted so that the optical components fill, in both cases, their Roche lobes. The pictures corresponds to the case of edge-on $\left(i=90^{0}\right)$ orientation of the binary orbit. Both light curves were computed by Dymitrow [3].

As one can see, the amplitude of the variability is so large ( $~ 0.5$ magnitudes) that, assuming random distribution of the orbital inclinations, the great majority of the systems should have amplitudes large enough to be detectable (even for $i=10^{\circ}$, the amplitude should be still $\sim 0.1$ magnitudes). Therefore, such systems should be easily detectable in mass photometric surveys.

We intend to start our search using catalogs of ASAS (All Sky Automated Survey) - a photometric survey carried out in the Astronomical Observatory of Warsaw University (Pojmański [7]). The limiting $V$ magnitude for ASAS survey is about 13, which for a typical system (mass of the optical component $\sim 1 \mathrm{M}_{\odot}$ ) translates into the limiting distance of about $0.5 \mathrm{kpc}$. This distance translates, in turn, into some 20 to 50 detectable BH transients (and some 40 to 300 detectable NS transients) if the critical value of the mass of the final compact object is $2 \mathrm{M}_{\odot}$. If this value is 3 $\mathrm{M}_{\odot}$, then the corresponding numbers are 1 to 8 detectable $\mathrm{BH}$ transients (and some 100 to 300 detectable NS transients).

Using the most likely set of assumptions $\left(M_{\mathrm{cr}}=2 \mathrm{M}_{\odot}\right.$, no kick during $\mathrm{BH}$ formation), we would expect some 20 detectable $\mathrm{BH}$ transients and some 200 detectable NS transients in the ASAS catalogs.

\section{Acknowledgments}

This work was partially supported by the State Committee for Scientific Research grants No 4 T12E 04727 and 1 P03D 01128.

\section{References}

[1] K. Belczyński, V. Kalogera \& T. Bulik, Binary Population Synthesis: Methods, Normalization, and Surprises, ApJ 572 (2002) 407 [astro-ph / 010222 ]]. 
[2] K. Belczyński, V. Kalogera, F.A. Rasio, R.E. Taam, A. Zezas, T. Bulik, T.J. Maccarone \& N. Ivanova, Compact Object Modeling with the StarTrack Population Synthesis Code, ApJ Suppl, (2006) in press, [astro-ph/051181].

[3] W. Dymitrow, Private communication (2006).

[4] Q.Z. Liu, J. van Paradijs \& E.P.J. van den Heuvel, A catalogue of high-mass X-ray binaries, $A \& A$ Suppl. 147 (2000) 25.

[5] Q.Z. Liu, J. van Paradijs \& E.P.J. van den Heuvel, A catalogue of low-mass X-ray binaries, A\&A 368 (2001) 1021.

[6] I.F. Mirabel \& I. Rodrigues, Formation of a black hole in the dark, Science 300 (2003) 1119.

[7] G. Pojmański, The All Sky Automated Survey, Acta Astr. 47 (1997) 467.

[8] J. Ziółkowski, Galactic Compact Objects, Frontier Objects in Astrophysics and Particle Physics (Procs. of the Vulcano Workshop 2004), eds. F. Giovannelli \& G. Mannocchi, Conference Proceedings, Italian Physical Society, Editrice Compositori, Bologna, Italy, 90 (2005) 335 [astro-ph/0502124]. 